\title{
Effect of Prognosis Awareness on the Survival and Quality of Life of Terminally III Cancer Patients: A Prospective Cohort Study
}

\author{
Hanna Lee ${ }^{1,2}$, Hae-Jin $\mathrm{Ko}^{1,2, *}$, A-Sol Kim ${ }^{1,3}$, Sung-Min Kim ${ }^{1,2}$, Hana Moon ${ }^{1,3}$, Hye-In Choi ${ }^{1,2}$ \\ ${ }^{1}$ Department of Family Medicine, School of Medicine, Kyungpook National University, Daegu, Korea \\ ${ }^{2}$ Department of Family Medicine, Kyungpook National University Hospital, Daegu, Korea \\ ${ }^{3}$ Department of Family Medicine, Kyungpook National University Chilgok Hospital, Daegu, Korea
}

Background: Physicians and caregivers are conflicted over whether to inform patients that their disease is terminal. Studies examining the effect of awareness of prognosis on the survival and quality of life of terminally ill cancer patients report conflicting results. This study aimed to assess the effects of prognosis awareness on the survival time and psychological health of terminally ill cancer patients.

Methods: Patients in the hospice wards of two general hospitals were asked to complete a questionnaire. All were mentally alert and could express themselves clearly. Awareness of prognosis was defined as knowing both the diagnosis and exact prognosis. Survival time was defined as the time from hospital admission to death. Multiple psychological examinations were conducted to verify the effect of prognosis awareness on psychological health.

Results: Of the 98 subjects who met the inclusion criteria, 65 (66.3\%) were aware of their terminal status. The patients' awareness was significantly related to survival time after adjusting for clinical variables with a hazard ratio of 1.70 (95\% confidence interval [CI], 1.01-2.86). Furthermore, the unaware group had a higher risk of cognitive impairment (Mini-Mental State Examination <24; adjusted odds ratio [aOR], 3.65; 95\% CI, 1.26-10.59) and a poorer quality of life (physical component summary of the Short Form 36-item Health Survey<20; aOR, 3.61; 95\% CI, 1.1211.60) than the aware group.

Conclusion: Knowledge of the exact prognosis might have a positive effect on the survival and quality of life of terminally ill cancer patients.

Keywords: Awareness; Hospices; Life Expectancy; Prognosis; Quality of Life

Received: July 20, 2018, Revised: August 29, 2018, Accepted: September 10, 2018

*Corresponding Author: Hae-Jin Ko https://orcid.org/0000-0003-4460-1476

Tel: +82-53-200-6578, Fax: +82-53-200-5480, E-mail: liveforme@knu.ac.kr 


\section{INTRODUCTION}

The increasing prevalence of cancer is accompanied by a concurrent increase in the need for hospice care. Studies conducted before 1970 showed that, in most cases, physicians did not inform patients of a terminal cancer diagnosis because they considered it inappropriate and immoral. ${ }^{1)}$ However, recently, most physicians have accepted the ethical argument that patients have the right to be informed of their diagnosis. ${ }^{2)}$ A previous study reports that in 1990, the majority (81.8\%) of physicians in Korea agreed to inform patients of their prognosis, compared to the $18 \%$ that did so in $1982 .{ }^{3)}$

Being informed of their prognosis and having an accurate prediction of survival time are important issues for terminal cancer patients. Patients with advanced cancer tend to misunderstand their illness, overestimate their prognosis, and have unrealistic expectations with respect to palliative treatment. ${ }^{4)}$ Patients who overestimate their prognosis are more likely to pursue aggressive treatment as they approach end-of-life. ${ }^{5)}$ Yun et al. ${ }^{6)}$ reported that patients who are unaware of their terminal status are more likely to require intensive care unit than those under palliative care. Moreover, without fully understanding the prognosis, patients cannot make well-informed decisions about treatment and end-of-life care. ${ }^{7)}$ Most terminal cancer patients want to know their exact status and life expectancy; ${ }^{8)}$ however, in actual clinical situations, physicians and families are still cconflicted over whether to inform patients of a terminal prognosis. ${ }^{9}$

Previous studies that examined the effect of prognosis awareness on terminally ill cancer patients analyzed their quality of life. These studies report conflicting results. ${ }^{10,11)}$ Furthermore, to the best of our knowledge, few studies have examined the relationship between patient awareness of a terminal prognosis and survival time; therefore, the results remain inconclusive. ${ }^{6,12)}$

Thus, we conducted a prospective cohort study to examine whether the awareness of disease diagnosis and prognosis affects not only quality of life, but also the life expectancy of terminally ill cancer patients.

\section{METHODS}

\section{Study Design and Subjects}

This prospective cohort study enrolled patients who were diagnosed with terminal cancer and admitted to the hospice ward of two general hospitals in Daegu, Korea, between March 2011 and September 2012. Patients were eligible to participate if they were at least 18-years-old, diagnosed with terminal cancer by their physician, capable of completing questionnaires or communicating with an interviewer, and had provided informed consent. Terminal cancer patients were defined as those with a progressive and advanced malignant disease who, from their physician's judgment, had a short life expectancy of a few months due to general prostration, refusal of further chemotherapy, or non-responsiveness to conventional anticancer therapy despite having undergone active treatment. Patients were not eligible to par- ticipate if they had other psychological diseases (e.g., depression or schizophrenia), had continued conventional anticancer treatment after enrollment, could not complete the questionnaire due to their physical or mental condition, or died within one day of admission.

All patients were required to provide informed consent to participate. This study was approved by the Institutional Review Board of the Kyungpook National University Chilgok Hospital, Daegu, Korea (Protocol no., KNUMC_13-1032).

\section{Definition of Awareness of the Terminal Status}

Patients' awareness of their terminal status was assessed through a semi-structured interview conducted by the family physicians in charge of the patients. Awareness of terminal status was defined as the patients understanding that conventional anticancer therapy was no longer curative for their illness or likely to extend their life significantly; their life expectancy was limited to several months. They also knew their disease diagnosis. Based on this definition, patients diagnosed with terminal cancer and admitted to the hospice ward were divided into two groups: "aware" subjects who knew the exact diagnosis and prognosis (i.e., terminal status) and "unaware" subjects who did not know their prognosis, irrespective of knowing the diagnosis.

\section{Demographic and Clinical Characteristics}

Patient information was collected within one or two days of admission and included age, gender, marital status, education, primary cancer site, presence of distant metastasis, previous interventions, use and dose of opioids (morphine or fentanyl), and laboratory results. The intensity of pain was assessed using the numerical rating scale (NRS; from 0 to 10): pain intensity reported as mild, moderate, or severe corresponded to NRS scores of $\leq 4,5-6$, and $\geq 7$, respectively. ${ }^{13)}$ Their Karnofsky performance status (KPS), ${ }^{14)}$ which is widely used to measure the physical function of cancer patients, was also assessed. In the palliative care setting, functional status assessments, such as the KPS, are used as a proxy indicator of quality of life and can guide decisions about palliative care.

\section{Primary Endpoint: Survival Time}

The primary outcome was the effect of the patient's unawareness of their disease prognosis on survival time. Survival time was defined as the interval (days) from the day of admission to death. The day of death was the date recorded on the death certificate.

\section{Secondary Endpoint: Psychological Assessment}

While hospitalized, the family medicine physician working in the hospice ward interviewed the patients and examined their psychological state using the following test tools.

\section{1) Depression scale (Beck Depression Inventory)}

The depression scale devised by Beck et al. ${ }^{15)}$ is used as a screening test and is generally suitable for clinical application. The Korean version of the scale, translated by Young Ho Lee and Jong Yong Song, was admin- 
istered in the form of a questionnaire. The scores of each of the 21 items, which ranged from 0 to 3 , were summed and classified. ${ }^{16)}$

\section{2) Anxiety scale (Beck Anxiety Inventory)}

The Beck Anxiety Inventory (BAI) identifies anxiety symptoms in patients with depression. It is composed of 21 items, with the total score ranging from 0 to 63 points. Each item is scored from 0 to 3 , with a higher score indicating a greater degree of anxiety. A score of 22 to 26 indicates anxiety, a score of 27 o 31 indicates severe anxiety, and a score of 32 and above indicates extreme anxiety. ${ }^{17)}$

\section{3) Health-Related Quality of Life Scale (36-item Short Form Health Survey)}

The 36-item Short Form Health Survey (SF-36) is a self-assessment questionnaire with 36 items. The physical component summary (PCS) is defined as the mean value of the four physical components (physical functioning $[\mathrm{PF}]$, role-physical [RP], bodily pain, and general health). The mental component summary is defined as the mean value of the four mental components (vitality, social functioning [SF], role-emo-

Table 1. Patient characteristics according to "awareness"

\begin{tabular}{|c|c|c|c|}
\hline Characteristic & Aware $(n=65)$ & Unaware $(n=33)$ & P-value \\
\hline Age (y) & $62.49 \pm 11.10$ & $68.76 \pm 14.53$ & 0.020 \\
\hline Sex (male) & $46(70.8)$ & $27(81.8)$ & 0.236 \\
\hline Marital status & & & 0.830 \\
\hline Married & $52(80.0)$ & 27 (81.8) & \\
\hline Other & $13(20.0)$ & 6 (18.2) & \\
\hline Educational attainment & & & 0.036 \\
\hline SElementary & $22(33.8)$ & $14(42.4)$ & \\
\hline Middle school & 9 (13.8) & $10(30.3)$ & \\
\hline zHigh school & $34(52.3)$ & $9(27.3)$ & \\
\hline Primary cancer site & & & $<0.001^{*}$ \\
\hline Gastrointestinal & $17(26.2)$ & $7(21.2)$ & \\
\hline Lung & $5(7.7)$ & $13(39.4)$ & \\
\hline Hepatobiliary & 8 (12.3) & $6(18.2)$ & \\
\hline Prostate & $12(18.5)$ & $0(0)$ & \\
\hline Breast/cervical & $3(4.6)$ & $3(9.1)$ & \\
\hline Other & $20(30.8)$ & $4(12.1)$ & \\
\hline Distant metastasis (present) & $62(95.4)$ & $26(78.8)$ & 0.010 \\
\hline \multicolumn{4}{|l|}{ Intervention } \\
\hline Operation & $29(44.6)$ & 7 (21.2) & 0.023 \\
\hline Chemotherapy & 47 (72.3) & $18(54.5)$ & 0.079 \\
\hline Radiotherapy & $22(33.8)$ & 5 (15.2) & 0.050 \\
\hline Numerical rating scale (pain) & $2.48 \pm 1.63$ & $3.30 \pm 2.08$ & 0.034 \\
\hline Karnofsky performance status & $42.15 \pm 16.72$ & $44.55 \pm 22.09$ & 0.587 \\
\hline \multicolumn{4}{|l|}{ Morphine } \\
\hline Usage & $41(63.1)$ & $24(72.7)$ & 0.339 \\
\hline Dose (mg oral morphine equivalents/d) & $83.68 \pm 186.62$ & $50.83 \pm 101.07$ & 0.430 \\
\hline \multicolumn{4}{|l|}{ Fentanyl } \\
\hline Usage & $51(78.5)$ & $26(78.8)$ & 0.970 \\
\hline Dose (mcg/d) & $109.28 \pm 178.21$ & $79.71 \pm 90.00$ & 0.430 \\
\hline
\end{tabular}

Values are presented as mean \pm standard deviation or number (\%). P-values were calculated using an independent t-test (continuous variables) and Pearson's chisquare test (categorical variables).

*By Fisher's exact test. tional, and mental health). This study used the Korean version of the scale translated by Han et al. ${ }^{18)}$

\section{4) Insomnia Scale (Insomnia Severity Index)}

The Insomnia Severity Index (ISI) is self-administered and used to assess insomnia. It is composed of seven items, each of which is rated on a 5-point Likert scale; the total score ranges from 0 to $28 .{ }^{19)}$

\section{5) Cognitive function scale (Mini-mental State Examination)}

The Mini-mental State Examination (MMSE) is the most commonly used screening tool to measure cognitive decline, with scores ranging from 0 to 30: a score of 24-30 indicates no cognitive impairment and a score of 18-23 indicates mild cognitive impairment. ${ }^{20)}$

\section{Statistical Analysis}

An independent t-test for continuous variables and Pearson's chisquare test for categorical variables were performed to compare the characteristics between groups. Survival analysis was performed using a multivariate Cox proportional-hazards regression model. Logistic regression analysis was performed to determine the effect of prognosis awareness on psychological status. All statistical analyses were performed using IBM SPSS Windows ver. 22.0 (IBM Corp., Armonk, NY, USA), where a P-value $<0.05$ was considered significant.

\section{RESULTS}

\section{Patient Characteristics}

These are summarized in Table 1 . Of the 98 patients, 33 were classified as "unaware" and 65 as "aware". The mean age of the unaware and the aware groups was 68.76 and 62.49 years, respectively $(\mathrm{P}=0.020)$. The primary cancer site (stomach, lung, liver biliary system, breast, cervix,

Table 2. Laboratory test results of the aware and unaware groups

\begin{tabular}{lccc}
\hline \multicolumn{1}{c}{ Variable } & Aware $(\mathrm{n}=65)$ & Unaware $(\mathrm{n}=33)$ & P-value \\
\hline White blood cell $\left(\times 10^{3} / \mathrm{mm}^{3}\right)$ & $10.30 \pm 6.29$ & $8.89 \pm 3.73$ & 0.169 \\
Hemoglobin $(\mathrm{g} / \mathrm{dL})$ & $10.39 \pm 1.77$ & $11.16 \pm 2.32$ & 0.073 \\
Platelets $\left(\times 10^{3} / \mu \mathrm{L}\right)$ & $257.20 \pm 141.42$ & $269.97 \pm 121.93$ & 0.644 \\
Glucose $(\mathrm{mg} / \mathrm{dL})$ & $137.99 \pm 62.46$ & $145.67 \pm 64.32$ & 0.570 \\
Creatinine $(\mathrm{mg} / \mathrm{dL})$ & $1.13 \pm 0.68$ & $1.50 \pm 1.08$ & 0.074 \\
Albumin $(\mathrm{g} / \mathrm{dL})$ & $3.63 \pm 2.58$ & $3.13 \pm 0.64$ & 0.279 \\
Total bilirubin $(\mathrm{mg} / \mathrm{dL})$ & $1.32 \pm 2.04$ & $2.09 \pm 4.68$ & 0.372 \\
Serum glutamic oxaloacetic & $58.68 \pm 115.51$ & $56.82 \pm 126.64$ & 0.942 \\
$\quad$ transaminase $(\mathrm{U} / \mathrm{L})$ & & & \\
Serum glutamic pyruvate & $28.62 \pm 34.04$ & $25.06 \pm 37.07$ & 0.637 \\
$\quad$ transaminase $(\mathrm{U} / \mathrm{L})$ & & & \\
Prothrombin time $(\mathrm{s})$ & $12.30 \pm 1.62$ & $18.67 \pm 26.69$ & 0.181 \\
Activated partial & $30.41 \pm 6.69$ & $28.64 \pm 6.13$ & 0.207 \\
$\quad$ thromboplastin time $(\mathrm{s})$ & & & \\
Calcium $(\mathrm{mg} / \mathrm{dL})$ & $9.17 \pm 0.95$ & $9.49 \pm 1.34$ & 0.174 \\
Sodium $(\mathrm{mmol} / \mathrm{L})$ & $132.76 \pm 4.75$ & $134.00 \pm 5.95$ & 0.263 \\
Potassium (mmol/L) & $4.20 \pm 0.66$ & $4.19 \pm 0.52$ & 0.938 \\
\hline
\end{tabular}

Values are presented as mean \pm standard deviation. P-values were calculated using an independent t-test. 
and prostate) was evenly distributed between the groups. Eighty-eight patients experienced distant metastasis to more than one site. The aware group experienced significantly less pain (score, 2.48) than the unaware group (score, 3.30) $(\mathrm{P}=0.034)$. There were no significant differences between the two groups in terms of the laboratory test results (complete blood cell counts, creatinine, glucose, albumin, total bilirubin, serum glutamic oxaloacetic transaminase, serum glutamic pyruvate transaminase, prothrombin time, activated partial thromboplastin time, and electrolyte levels) known to be associated with prognosis. The results are shown in Table 2.

\section{Differences in Psychoanalytic Scale Scores between the Aware and Unaware Groups}

The Beck Depression Inventory (BDI), BAI, ISI, MMSE, and SF-36 scores of the unaware and aware groups were compared (Table 3 ). The mean BDI for the unaware and aware groups was 25.94 and 29.43, respectively, which exceeded the depressive threshold of 22, but was not statistically significant. The mean BAI was 24.94 and 28.11, respectively. There was no difference in the anxiety score $(\mathrm{P}=0.29)$. The ISI for the unaware and aware groups was 13.70 and 13.79, respectively, which indicates subthreshold insomnia (ISI, 8-14); however, the difference between the groups was not statistically significant. The mean MMSE score of the unaware group was 21.94 and that of the aware group was 25.45; thus, cognitive function was significantly better in the aware group $(\mathrm{P}=0.003)$.

The results of the SF-36 showed a significant association with awareness. The PF, SF, RP, and PCS scores of the aware group were significantly higher than those of the unaware group (PF: 31.71 versus 22.83, $\mathrm{P}=0.001$; $\mathrm{SF}: 34.09$ versus 27.69, $\mathrm{P}=0.011$; $\mathrm{RP}: 32.37$ versus 29.27, $\mathrm{P}=0.004$; and PCS: 31.24 versus 25.64, $\mathrm{P}=0.002$, respectively).

\section{Primary Endpoint: Effect of Unawareness of Disease Prognosis on Survival}

Patient awareness was a significant factor for survival. After adjusting for clinical variables (age, gender, primary cancer site, distant metastasis status, and NRS), the unaware group had a shorter survival period than the aware group (hazard ratio, 1.70; 95\% confidence interval [CI], 1.01-2.86; P=0.046) (Figure 1).
Table 3. Psychometric test results of the aware and unaware groups

\begin{tabular}{llll}
\hline \multicolumn{1}{c}{ Variable } & \multicolumn{1}{c}{$\begin{array}{c}\text { Aware } \\
(\mathrm{n}=65)\end{array}$} & $\begin{array}{c}\text { Unaware } \\
(\mathrm{n}=33)\end{array}$ & P-value \\
\hline Beck Depression Inventory (depression) & $29.43 \pm 11.26$ & $25.94 \pm 11.15$ & 0.149 \\
Beck Anxiety Inventory (anxiety) & $28.11 \pm 14.84$ & $24.94 \pm 12.07$ & 0.291 \\
Insomnia Severity Index (insomnia) & $13.79 \pm 8.35$ & $13.70 \pm 9.18$ & 0.962 \\
Mini-mental State Examination & $25.45 \pm 3.04$ & $21.94 \pm 6.01$ & 0.003 \\
$\quad$ (cognitive function) & & & \\
36-Item Short Form Health Survey (quality of life) & & \\
Total & $31.48 \pm 6.77$ & $29.28 \pm 5.00$ & 0.073 \\
Physical functioning & $31.71 \pm 14.21$ & $22.83 \pm 10.12$ & 0.001 \\
Social functioning & $34.09 \pm 14.03$ & $27.69 \pm 9.97$ & 0.011 \\
Role-physical & $32.37 \pm 7.47$ & $29.27 \pm 2.74$ & 0.004 \\
$\quad$ Role-emotional & $29.65 \pm 9.10$ & $26.91 \pm 9.65$ & 0.171 \\
$\quad$ Mental health & $30.05 \pm 9.36$ & $30.36 \pm 9.60$ & 0.883 \\
Vitality & $33.30 \pm 9.60$ & $35.15 \pm 8.47$ & 0.398 \\
Bodily pain & $39.03 \pm 10.42$ & $34.91 \pm 11.34$ & 0.076 \\
$\quad$ General health & $24.54 \pm 8.35$ & $26.29 \pm 6.25$ & 0.289 \\
$\quad$ Physical component summary & $31.24 \pm 8.60$ & $25.64 \pm 7.13$ & 0.002 \\
$\quad$ Mental component summary & $31.72 \pm 8.47$ & $32.92 \pm 8.05$ & 0.501 \\
\hline
\end{tabular}

Values are presented as mean \pm standard deviation. P-values were calculated using an independent t-test.

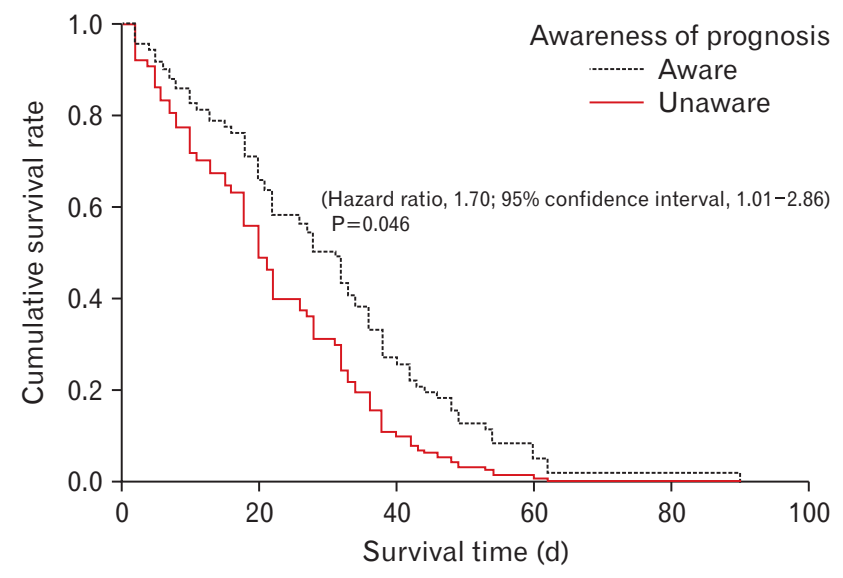

Figure 1. Survival analysis of the effect of unawareness of disease prognosis on survival time. Multivariate Cox regression analysis of the cumulative survival time of terminally ill cancer patients according to their awareness of their prognosis (adjusted for age, gender, primary cancer site, distant metastasis status, and numeric rating scale).

Table 4. Relationship between awareness of terminal status and psychological status

\begin{tabular}{llcc}
\hline \multicolumn{1}{c}{ Psychological status } & \multicolumn{1}{c}{ Psychometric tests } & Adjusted odds ratio (95\% confidence interval) & P-value \\
\hline Depression & Beck Depression Inventory $\geq 24$ & $0.653(0.173-2.462)$ & 0.529 \\
Anxiety & Beck Anxiety Inventory $\geq 22$ & $0.785(0.309-1.999)$ & 0.612 \\
Insomnia & Insomnia Severity Index $\geq 15$ & $0.830(0.334-2.063)$ & 0.689 \\
Cognitive impairment & Mini-mental State Examination $<24$ & $3.654(1.260-10.596)$ & 0.017 \\
Poor quality of life & Physical component summary $<20$ & $3.614(1.126-11.601)$ & - \\
& Mental component summary $<17$ & - & - \\
Pain & Numerical rating scale $\geq 4$ & $2.110(0.811-5.491)$ & 0.126
\end{tabular}

P-values were calculated using logistic regression analysis, with the awareness group as the independent variable (model adjusted for age, primary cancer site, and educational attainment). The aware group was used as the reference. 


\section{Secondary Endpoint: Relationship between Awareness of Disease Prognosis and Psychological Status}

Logistic regression analyses (adjusted for age, primary cancer site, and educational attainment) was performed to examine the effect of disease prognosis awareness on psychological status. The unaware group had a higher risk of cognitive impairment (adjusted odds ratio [aOR], 3.65 ; 95\% CI, 1.26-10.59) and a poorer quality of life (aOR, 3.61; 95\% CI, 1.12-11.60) than the aware group. There was no significant association between awareness and the results of other psychometric tests (Table 4).

\section{DISCUSSION}

To the best of our knowledge, few studies have examined the effect of awareness of an exact prognosis on the survival time of terminally ill cancer patients. We found that patients who were aware of their prognosis and diagnosis lived longer than those who were not aware; this finding was consistent after adjusting for several covariates that could influence the survival of cancer patients. Even though the majority of cancer patients want to know their status and life expectancy, ${ }^{8)}$ cultural differences in the attitude of physicians and caregivers leads to the belief that bad news is not always given to patients. ${ }^{21)}$ In particular, caregivers in Asian countries are less likely to inform patients of their actual prognosis than those in Western countries. ${ }^{21,22)}$ For this reason, we believe that the results of this study are interesting because they suggest that awareness of terminal status improves survival time. However, a previous study conducted in Korea reported better survival and quality of life in patients that were unaware. ${ }^{12)}$ There are several reasons for these opposing results. First, we did not adjust for performance status during survival analysis because we found no significant difference in performance status between the two groups. The previous study adjusted for different factors (age, educational level, primary cancer site, time since cancer diagnosis, Montgomery-Asberg Depression Rating Scale score, and Eastern Cooperative Oncology Group Performance Status), which could explain the contradictory results. Moreover, a univariate analysis conducted in the previous study showed that the awareness of terminal status itself did not affect overall survival $(\mathrm{P}=0.132)$. Second, the distribution of primary cancer sites was different. Here, the incidence of lung cancer (which has a relatively low survival rate) in the unaware group was relatively low. However, the previous study reported a higher incidence of head, neck, and pancreatic cancers in the aware group; all of these cancers have relatively short median survival times. Third, the difference in the mean age of the aware and unaware groups was greater in the previous study than in our own (9.0 versus 6.26 years, respectively). These might have contributed to the contradictory results.

Considering that an MMSE score below 24 suggests cognitive impairment, the difference between the two groups is meaningful. We adjusted for age because the unaware group was older than the aware group; however, the difference remained significant. Patients who did not know their exact diagnosis and prognosis experienced a signifi- cantly greater decline in cognitive function. Several underlying mechanisms have been proposed, including the biology of the cancer itself and the risk factors underlying cancer development and cognitive decline. ${ }^{23)}$ Recently, a non-central nervous system tumor was found to be the factor that triggered hippocampal dysfunction, possibly by reducing the rate of neurogenesis and levels of brain-derived neurotrophic factor and cyclooxygenase 2, in addition to increasing stress-related parameters and circulating levels of proinflammatory cytokines. ${ }^{24)}$ However, the pathophysiology of cognitive impairment is still unclear; conversely, it is possible that patients with better cognitive function are able to remember their prognosis. Furthermore, the causality is unclear because the cognitive function test was conducted only one time. Further studies with serial psychometric tests would be needed.

Previous studies on awareness of prognosis and quality of life reached different conclusions. ${ }^{10)}$ Although some studies suggest that a diagnosis of cancer and prognosis awareness increase anxiety and depression, thereby reducing physical, social, and emotional quality of life. ${ }^{25)}$ Others suggest that depression increases more than threefold when the patient does not know the prognosis; indeed, neuropsychological stress and emotional disturbances are more common in such cases. ${ }^{26)}$ Other studies report no association. ${ }^{27)}$ Here, the SF-36 score for assessing quality of life showed that, after adjusting for age, the unaware group had a 3.61-fold higher risk of reporting a poorer healthrelated quality of life score (PCS $<20$ ); however, there was no significant difference between the groups in terms of depression, anxiety, or insomnia. This is contrary to the common belief that quality of life deteriorates when a patient is aware of the prognosis. ${ }^{6)}$ This conclusion is consistent with those of previous studies that report no significant relationship between anxiety, depression, and patient-to-physician relationships before and after counseling. ${ }^{28)}$ These inconsistent conclusions with regard to the quality of life of patients with terminal cancer suggest that it is not determined by one simple factor. Moreover, it is not only psychosocial factors, such as individual attitudes to life, that have an effect; other physical factors such as the presence or absence of distant metastasis and different symptoms according to the primary cancer site also play a role. ${ }^{29)}$ Tang et al. ${ }^{30}$ identified the factors that affect quality of life. They then evaluated quality of life by reviewing appropriate observations, interventions, and introducing programs that could help patients. They also provided opportunities for them to prepare for death.

\section{Study Limitations}

This study has several limitations. First, the subjects were relatively old and lived in an urban province and patients who had difficulty communicating or withdrew consent were excluded. Therefore, we cannot rule out selection bias. Second, the number of participants included in the final analysis was small, and the timing of prognosis awareness is uncertain; thus, the results may not be generalizable. Third, the population was Korean; this suggests that the results may be ethnically or geographically restricted. Also, it should be noted that the reported differences in attitudes toward disclosure (compared with other ethnic 
groups) suggest that ethnic-specific evaluations may be required. ${ }^{22)}$ Fourth, we did not consider the possibility of denial, which is one stage of adapting to a terminal disease. A previous study suggests that denial is an important variable that results in false beliefs about prognosis and treatment goals. ${ }^{31)}$ Fifth, the SF-36 questionnaire, which was used in evaluating the participants, was designed to assess quality of life of healthy persons. In the future, it might be useful to compare quality of life with other symptoms using measures developed specifically for patients receiving palliative care.

\section{Clinical Implications}

However, this study enrolled patients with terminal cancer who were residents in hospitals with hospice wards; therefore, it is advantageous to use various psychometric assessments. In addition, it is important to confirm the association between survival rate and prognosis awareness. Our finding that awareness of terminal status is related to longer survival times and a better quality of life may have important implications for family caregivers and physicians who are reluctant to disclose a diagnosis of terminal illness, especially in Korea. In the future, largescale studies should be conducted in the hospice wards of various institutions in different regions.

\section{Conclusion}

We found that awareness of the prognosis of terminal cancer in a palliative care setting may have a positive effect on survival time and quality of life. This may help the patient lead a more meaningful life; therefore, we recommend that physicians adopt a more patient-oriented attitude in communication when discussing prognostic information.

\section{CONFLICT OF INTEREST}

No potential conflict of interest relevant to this article was reported.

\section{ORCID}

Hanna Lee: https://orcid.org/0000-0002-4845-0743

Hae-Jin Ko: https://orcid.org/0000-0003-4460-1476

A-Sol Kim: https://orcid.org/0000-0003-2497-9500

Sung-Min Kim: https://orcid.org/0000-0001-9942-6893

Hana Moon: https://orcid.org/0000-0003-4604-3746

Hye-In Choi: https://orcid.org/0000-0001-9438-8680

\section{REFERENCES}

1. Friedman HJ. Physician management of dying patients: an exploration. Psychiatry Med 1970;1:295-305.

2. Jenkins V, Fallowfield L, Saul J. Information needs of patients with cancer: results from a large study in UK cancer centres. Br J Cancer 2001;84:48-51.

3. Waltman NL. Attitudes, subjective norms, and behavioral intentions of nurses toward dying patients and their families. Oncol Nurs Forum
1990;17(3 Suppl):55-62.

4. Chow E, Andersson L, Wong R, Vachon M, Hruby G, Franssen E, et al. Patients with advanced cancer: a survey of the understanding of their illness and expectations from palliative radiotherapy for symptomatic metastases. Clin Oncol (R Coll Radiol) 2001;13:204-8.

5. Matsuyama R, Reddy S, Smith TJ. Why do patients choose chemotherapy near the end of life?: a review of the perspective of those facing death from cancer. J Clin Oncol 2006;24:3490-6.

6. Yun YH, Lee MK, Kim SY, Lee WJ, Jung KH, Do YR, et al. Impact of awareness of terminal illness and use of palliative care or intensive care unit on the survival of terminally ill patients with cancer: prospective cohort study. J Clin Oncol 2011;29:2474-80.

7. Ghandourh WA. Palliative care in cancer: managing patients' expectations. J Med Radiat Sci 2016;63:242-57.

8. Yun YH, Lee CG, Kim SY, Lee SW, Heo DS, Kim JS, et al. The attitudes of cancer patients and their families toward the disclosure of terminal illness. J Clin Oncol 2004;22:307-14.

9. Gordon EJ, Daugherty CK. 'Hitting you over the head': oncologists' disclosure of prognosis to advanced cancer patients. Bioethics 2003;17:142-68.

10. Fan X, Huang H, Luo Q, Zhou J, Tan G, Yong N. Quality of life in Chinese home-based advanced cancer patients: does awareness of cancer diagnosis matter? J Palliat Med 2011;14:1104-8.

11. Lee MK, Baek SK, Kim SY, Heo DS, Yun YH, Park SR, et al. Awareness of incurable cancer status and health-related quality of life among advanced cancer patients: a prospective cohort study. Palliat Med 2013;27:144-54.

12. Kim SY, Kim JM, Kim SW, Shin IS, Bae KY, Shim HJ, et al. Does awareness of terminal status influence survival and quality of life in terminally ill cancer patients? Psychooncology 2013;22:2206-13.

13. Ripamonti CI, Santini D, Maranzano E, Berti M, Roila F; ESMO Guidelines Working Group. Management of cancer pain: ESMO Clinical Practice Guidelines. Ann Oncol 2012;23 Suppl 7:vii139-54.

14. Karnofsky DA, Abelmann WH, Craver LF, Burchenal, JH. The use of the nitrogen mustards in the palliative treatment of carcinoma: with particular reference to bronchogenic carcinoma. Cancer 1948;1:63456.

15. Beck AT, Ward CH, Mendelson M, Mock J, Erbaugh J. An inventory for measuring depression. Arch Gen Psychiatry 1961;4:561-71.

16. Wang YP, Gorenstein C. Assessment of depression in medical patients: a systematic review of the utility of the Beck Depression Inventory-II. Clinics (Sao Paulo) 2013;68:1274-87.

17. Beck AT, Epstein N, Brown G, Steer RA. An inventory for measuring clinical anxiety: psychometric properties. J Consult Clin Psychol 1988;56:893-7.

18. Han CW, Lee EJ, Iwaya T, Kataoka H, Kohzuki M. Development of the Korean version of Short-Form 36-item Health Survey: health related QOL of healthy elderly people and elderly patients in Korea. Tohoku J Exp Med 2004;203:189-94.

19. Bastien CH, Vallieres A, Morin CM. Validation of the Insomnia Severity Index as an outcome measure for insomnia research. Sleep Med 2001;2:297-307.

20. Park JH. Standardization of Korean version of the Mini-Mental State Examination (MMSE-K) for use in the elderly: part II. diagnostic validity. J Korean Neuropsychiatr Assoc 1989;28:508-13. 
21. Blackhall LJ, Murphy ST, Frank G, Michel V, Azen S. Ethnicity and attitudes toward patient autonomy. JAMA 1995;274:820-5.

22. Fujimori M, Akechi T, Morita T, Inagaki M, Akizuki N, Sakano Y, et al. Preferences of cancer patients regarding the disclosure of bad news. Psychooncology 2007;16:573-81.

23. Seigers R, Fardell JE. Neurobiological basis of chemotherapy-induced cognitive impairment: a review of rodent research. Neurosci Biobehav Rev 2011;35:729-41.

24. Yang M, Kim J, Kim JS, Kim SH, Kim JC, Kang MJ, et al. Hippocampal dysfunctions in tumor-bearing mice. Brain Behav Immun 2014;36: 147-55.

25. Montazeri A, Tavoli A, Mohagheghi MA, Roshan R, Tavoli Z. Disclosure of cancer diagnosis and quality of life in cancer patients: should it be the same everywhere? BMC Cancer 2009;9:39.

26. Chochinov HM, Tataryn DJ, Wilson KG, Ennis M, Lander S. Prognostic awareness and the terminally ill. Psychosomatics 2000;41:500-4.
27. Iconomou G, Viha A, Koutras A, Vagenakis AG, Kalofonos HP. Information needs and awareness of diagnosis in patients with cancer receiving chemotherapy: a report from Greece. Palliat Med 2002;16:31521.

28. Enzinger AC, Zhang B, Schrag D, Prigerson HG. Outcomes of prognostic disclosure: associations with prognostic understanding, distress, and relationship with physician among patients with advanced cancer. J Clin Oncol 2015;33:3809-16.

29. Maeda T, Hayakawa T. Effectiveness of corticosteroid monotherapy for dyspnea relief in patients with terminal cancer. J Pain Palliat Care Pharmacother 2017;31:148-53.

30. Tang WR, Aaronson LS, Forbes SA. Quality of life in hospice patients with terminal illness. West J Nurs Res 2004;26:113-28.

31. Gattellari M, Butow PN, Tattersall MH, Dunn SM, MacLeod CA. Misunderstanding in cancer patients: why shoot the messenger? Ann Oncol 1999;10:39-46. 